\title{
A case study of electron precipitation in the late substorm growth phase on and nearby a preonset arc
}

\author{
A. Olsson ${ }^{1}$ and P. Janhunen ${ }^{2,1}$ \\ ${ }^{1}$ Swedish Institute of Space Physics, S-755 91 Uppsala, Sweden \\ ${ }^{2}$ Finnish Meteorological Institute, Helsinki, Finland
}

Received: 15 September 1997 / Accepted: 26 March 1998

\begin{abstract}
We follow the electron precipitation characteristics on and nearby a preonset arc using the high resolution Freja TESP instrument. Our data coverage extends from about $10 \mathrm{~min}$ before onset up to $1 \mathrm{~min}$ before onset. The arc is the most equatorward one (around MLAT $62^{\circ}$ ) of a system of growth phase arcs, and it was close to the radiation belt precipitation. Within the preonset arc, inverted-V type precipitation dominates. Poleward of the arc we also find some precipitation regions, and here there is systematically a cold electron population superposed with a warm population. Using single and double Maxwellian fits to the measured electron spectra we find the ionospheremagnetosphere coupling parameters (field-aligned conductance $K$ and the parallel potential drop $V$ ) as well as the effective source plasma properties (density and temperature) during the event. Compared to typical expansion phase features, the preonset parallel potential drop is smaller by a factor of ten, the electron temperature is smaller by a factor of at least five, and the field-aligned conductance is about the same or larger. The fact that there are two isotropic superposed electron populations on the poleward side of the preonset arc suggests that the distance between warm trapped electrons on dipolar field lines and colder electrons on open field lines has become so small near the onset that mixing e.g. due to finite electron Larmor radius effects can take place.
\end{abstract}

Key words. Ionosphere (ionosophere-magnetosphere interactions) · Magnetospheric physics (auroral phenomena; storms and substorms).

\section{Introduction}

The following scenario of a typical growth phase in the magnetosphere is today generally accepted (Elphinstone et al., 1996): a southward turning of the IMF $B_{z}$, and consequently a dayside reconnection resulting in enhanced convection directly driven by the solar wind. This will increase the strength of the magnetospheric currents (most importantly, region-1 and cross-tail current), and a loading of energy in the magnetotail will go on until the onset of the substorm, when a second current system (the substorm current wedge, SCW) due to unloading will develop.

Studies of flux-energy electron spectra can give us valuable clues of many magnetospheric properties and also give information of energization processes that the particles have undergone on the way to the ionosphere. Usually, three main categories of electron precipitation are considered (McFadden et al., 1987): diffuse precipitation, inverted- $\mathrm{V}$ precipitation, and field-aligned precipitation. The diffuse precipitation (Fig. 1a) is characterized by a Maxwellian distributed spectrum and is associated with diffuse aurora. The electrons probably precipitate from trapped regions or from the plasma sheet without any acceleration along the fieldline. The inverted-V-type events (Fig. 1b) are thought to result from acceleration through a parallel potential drop and they are often associated with discrete arcs (Frank and Ackerson, 1971). The peak electrons in the spectra, most likely of plasma sheet orgin, show an accelerated Maxwellian distribution (Evans, 1974). If the accelerating voltage is small, then the inverted-V spectrum becomes equal to diffuse precipitation. Fieldaligned electron fluxes are third category (Lin and Hoffman, 1979; Arnoldy et al., 1985). The strong beam collimation argues for the presence of a field-aligned acceleration process not far above the point of measurement. The presence of a field-aligned flux peak together with an isotropic flux may suggest the 


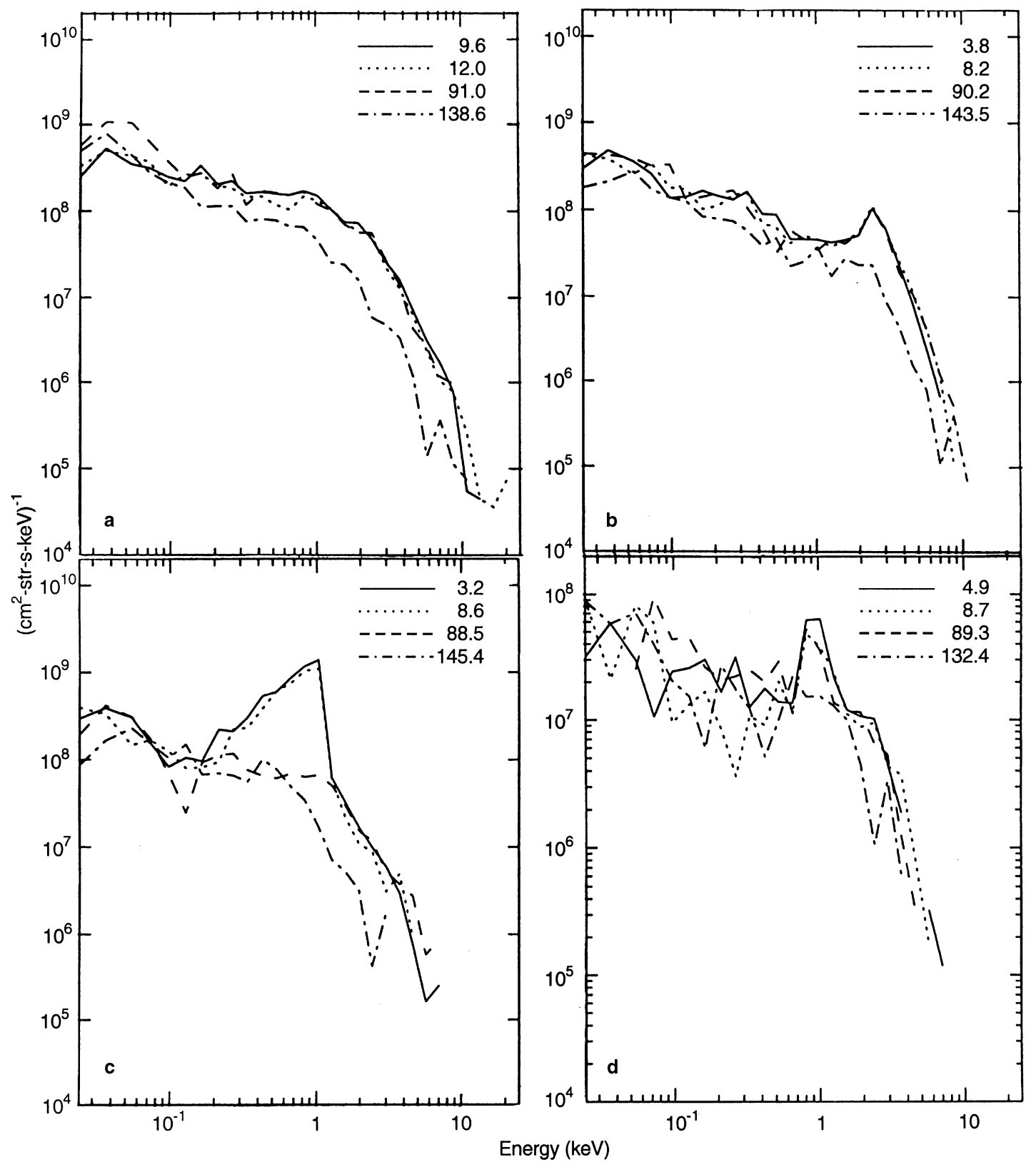

Fig. 1. Example particle flux spectra of a diffuse (21:02:38), b inverted-V (21:02:14), c field-aligned superposed with isotropic (21:01:48), d two isotropic inverted-V populations (21:04:22). Both populations have $V \approx 1 \mathrm{kV}$ but different characteristic energies around $200 \mathrm{eV}$ and $1 \mathrm{keV}$

existence of two separate potential drops (McFadden et al., 1990). However, field-aligned bursts can also be due to electrons accelerated by inertial Alfvén wave interaction (Goertz and Boswell, 1979; Hui and Seyler, 1992).

The main purpose of this work is to gain some quantitative estimates for the ionosphere-magnetosphere coupling parameters (the field-aligned conductance $K$ and the accelerating potential $V$ ) as well as for the effective source plasma properties (the density $n_{\text {src }}$ and the thermal energy (i.e. characteristic energy) $E_{c}$ ) in the geophysically interesting and well-defined case of the preonset arc in the late growth phase.

\section{Instrumentation}

The Swedish-German satellite Freja was launched in 1992 (Lundin et al., 1994). With the somewhat unusual inclination of $63^{\circ}$, Freja often passes tangentially along or crosses the auroral oval twice and thus gives us a possibility of following an arc during a longer period. In this study Freja crosses the preonset arc (Fig. 2) at an altitude of around $1750 \mathrm{~km}$, which means that the accelerating potential drop is most often entirely above Freja. The two-dimensional electron spectrometer (TESP) on the Freja F7 experiment (Boehm et al., 1994) measures simultaneously in 32 directions sweeping over 32 discrete energy levels between $20 \mathrm{eV}$ and $25 \mathrm{keV}$. 


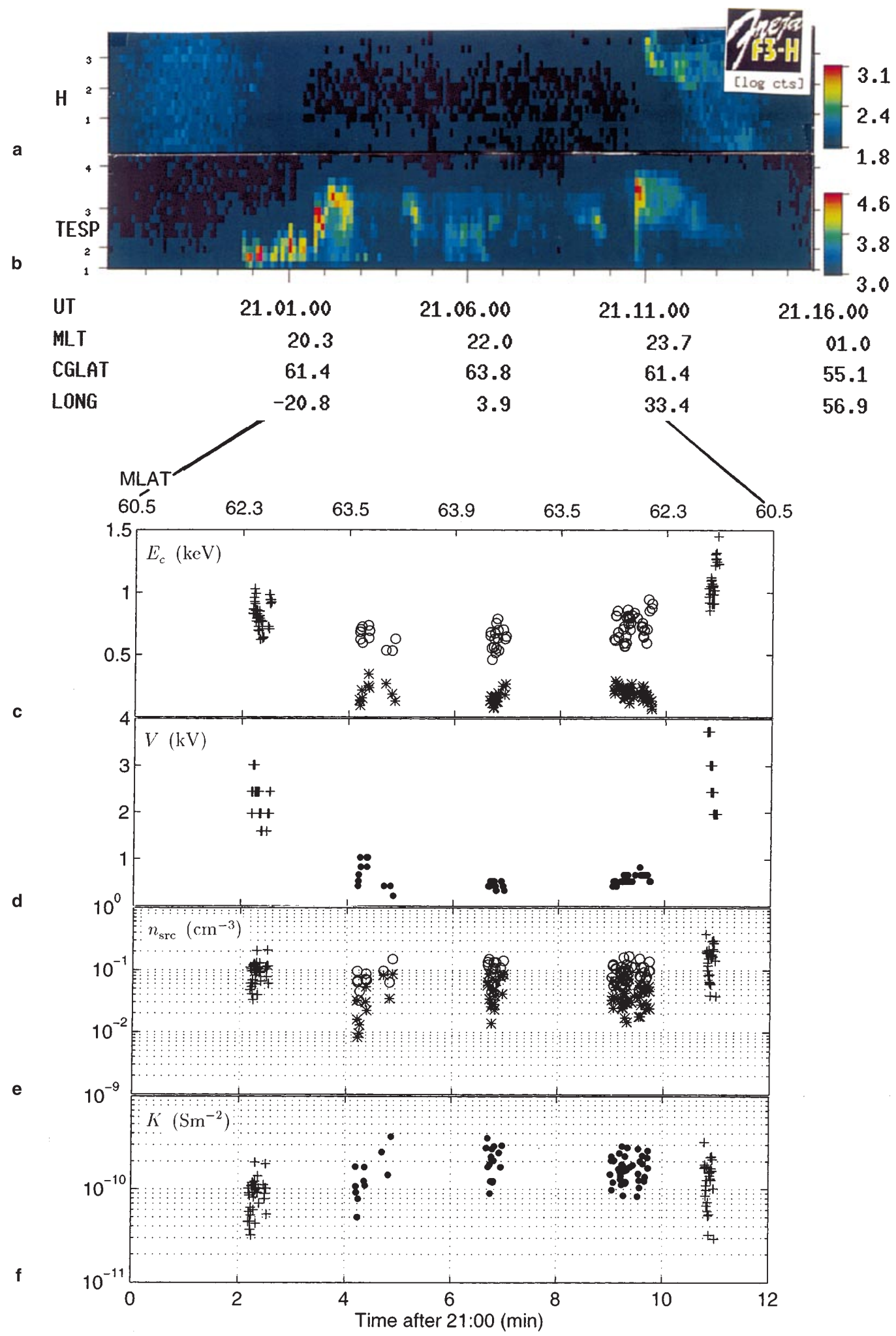

Fig. 2. a F3H hydrogen data showing the ring current regions around our event. b TESP electron energy flux spectrum. This is the raw data which Fig. 1 and the rest of the panels of Fig. 2 are based on. $\mathbf{c}$ Fitted characteristic (thermal) energy $E_{c}$ for preonset arc inverted-V precipitation $(+)$, poleward cold precipitation $(*)$, poleward warm precipitation (०). Data gaps are due to too little precipitation for reliable classification or otherwise failed fits. d Fitted accelerating potential $V$ for inverted-V precipitation over the preonset arc $(+)$ and combined warm/cold poleward precipitation (dots). e Effective source plasma density $\mathrm{n}_{\text {src }}$ with the same symbols as in c. $\mathbf{f}$ Field-aligned conductance $K$ with the same symbols as in panel d 
We also use the $\mathrm{F} 3 \mathrm{H}$ ion data to identify the ring current regions.

\section{Observations}

From LANL geosynchronous satellites, pulsation signatures, all-sky camera images and magnetograms Persson et al. (1994) verify that the onset on March 2 1993 takes place 2112 UT on the same preonset arc that is covered by Freja in the present study.

According to all-sky camera images (not shown here), the preonset arc is formed already around 2056:00 UT and stays stable until the start of the expansion phase. Freja enters the onset region about 10 min before the onset. Freja leaves the onset region $1 \mathrm{~min}$ before the onset.

\subsection{Characteristics of the spectra}

In this study all three categories mentioned in the Introduction are observed. Clearly identifiable diffuse precipitation is observed for about 16 s only (Fig. 1a). About half of our event consists of precipitation that is too weak to be reliably classified; it is conceivable that most of this weak precipitation is of the diffuse type. Inverted- $\mathrm{V}$ is the main type of precipitation observed in this event (Fig. 1b). Field-aligned precipitation is observed for about $20 \mathrm{~s}$ at the equatorward edge of the preonset arc. This field-aligned precipitation is, however, superposed with isotropic inverted-V precipitation (Fig. 1c). Poleward of the preonset arc we observe spectra that do not fit directly into the three categories mentioned, namely two superposed isotropic inverted-V spectra of two different characteristic energies (Fig. 1d). These populations have most probably been accelerated through a common field-aligned potential drop at a high altitude. We call these the warm and cold isotropic populations. The colder population temperature is $100-400 \mathrm{eV}$, so it is clearly of magnetospheric origin.

Figure 2a shows the $\mathrm{F} 3 \mathrm{H}$ hydrogen flux, which clearly indicates the ring current signatures on the equatorward side of the preonset arc. Fig. $2 b$ shows the TESP electron data to which all the fits in this work were carried out. The preonset arc passes are seen around 2101 and 2111 UT, respectively, and they mainly consist of inverted-V precipitation. Between those the more irregular poleward precipitation is observed, which mainly consists of two superposed isotropic inverted-V populations. The two clear precipitation gaps on the poleward edges of the preonset arc (2103-2104 and 2110 , respectively) might be due to downward currents closing the upward currents in the arcs.

\subsection{Estimates of $V, n_{s r c}, E_{c}$ and $K$}

To gain quantitative information on the preonset current-voltage relationship parameters, which are re- lated to source plasma properties, we fitted accelerated Maxwellian functions to the spectra (Evans, 1974). The fitted parameters were the source plasma density $n_{\text {src }}$, the accelerating voltage $V$ and the source plasma temperature (characteristic energy) $E_{c}$. The potential $V$ was determined from the position of the peak flux. Only good fits were accepted for further processing; the quality of the fits was confirmed manually for all data points.

In case of the poleward precipitation where we had clearly two superposed populations we fitted two accelerated Maxwellian distributions in adjacent, nonoverlapping energy ranges which were selected manually. In the fit a common potential drop $V$ which was also manually determined from the peak of the distribution was assumed for both distributions, this can be justified from the data in most cases since the data has only a single, rather narrow maximum.

Under the assumption of a linear current-voltage relationship (Lemaire and Scherer, 1983) we can, from these parameters, also estimate the field-aligned conductance $K$ and corresponding current density $j$ according to

$j=\frac{e^{2}}{(2 \pi m)^{1 / 2}} \frac{n_{\mathrm{src}}}{E_{c}^{1 / 2}} V \equiv K V$.

The resulting $E_{c}, V, n_{\mathrm{src}}$ and $K$ values are shown in Fig. 2c-f, where the parameters are plotted versus time and MLAT as Freja passes the auroral structures.

The source plasma densities are widely spread $(0.01-$ $0.1 \mathrm{~cm}^{-3}$ ) (Fig. 2e) and usually unrealistically low. The source density is not necessarily the same as the true magnetospheric plasma density, but it is probably less than that because the loss cone filling during one electron bounce period (Janhunen et al., 1995) may be incomplete. However, as we do not see a clear signature of the downward loss cone in our TESP data a direct evidence for this explanation is lacking.

In the poleward precipitation the two different populations show differences in characteristic energies (Fig. 2c; around 0.2 and $0.7 \mathrm{keV}$, respectively). The characteristic energies of the inverted- $\mathrm{V}$ populations in the preonset arc are in the range $0.7-1.3 \mathrm{keV}$.

The potential drop (Fig. 2d) for the poleward precipitation is low $(0.5 \mathrm{kV})$, while for the preonset arc it varies in the range $2-3 \mathrm{kV}$.

In Fig. $2 \mathrm{f}$ we have summed up the contributions to a total $K$ (Eq. 1) from the two populations in the poleward precipitation. The $K$ value for the poleward precipitation is around $1 \cdot 10^{-10}-1.2 \cdot 10^{-10} \mathrm{~S} / \mathrm{m}^{2}$, while it is slightly lower for the preonset arc precipitation. Notice that since we have two different source plasma populations, it is correct to compute $K$ as $K=K_{1}+K_{2}$ (the analog of two parallel resistors, recall that $K$ is inverse resistance). On the other hand, if we had two different $K$ values due to two different acceleration processes (the mirror force and anomalous resistivity, for instance), then the total $K$ value would be $K=1 /\left(1 / K_{1}+1 / K_{2}\right)$ (the analog of two serial resistors). 
Notice that Fig. 2d, f have the two poleward isotropic populations combined (black circles) whereas Fig. 2c, e have them separately (stars and open circles). The presonset arc inverted-V precipitation is shown by plus signs throughout.

The general trends seen in Fig. 2 (larger $E_{c}$ and $V$ over the preonset arc than elsewhere) occur naturally since the magnetospheric plasma temperature increases when one move towards the Earth and because higher potential drops are generally associated with arcs.

\section{Discussion}

We have studied a unique event where the low-altitude Freja satellite measures electron data during the late growth phase of a substorm. Freja enters the onset region about $10 \mathrm{~min}$ before the onset and passes over the preonset arc twice before leaving the onset region one minute before the onset. We have studied the precipitation characteristics both in the preonset arc itself, where inverted-V precipitation dominates, and in the region on the poleward side of the arc, where a cold $(0.1-0.3 \mathrm{keV})$ electron population is superposed to a warm population. It is possible to get quantitative estimates for the ionosphere-magnetosphere coupling parameters $K$ and $V$ as well as for the effective source plasma properties $n_{\text {src }}$ and $E_{c}$. These parameters may provide important information on the state of the magnetosphere as well as ionosphere-magnetosphere coupling just before the substorm onset.

The measured source densities are unrealistically low $\left(0.01-0.1 \mathrm{~cm}^{-3}\right)$. A reasonable explanation to this is incomplete loss cone filling (Janhunen, 1996), although we are unable to point to direct evidence for this explanation model in our data.

Compared to substorm expansion phase (Olsson et al., 1996), the electrons are colder (by a factor of five) and the potential drops much smaller, about 2-3 $\mathrm{kV}$. The field-aligned conductance $K$ is about $10^{-10} \mathrm{~S}$ $\mathrm{m}^{-2}$, which is significantly smaller than during quiet magnetospheric conditions and about the same or larger than in expansion phase. The fact that the potential drop may be up to ten times higher after the onset than just before is consistent with the appearance of bright auroral forms after onset since a high potential drop increases the ionospheric ionization production rate and conductivity efficiently.

The preonset arc is located on a low latitude (around mlat $62^{\circ}$ ), close to the radiation belt region. The inverted-V precipitation is the most dominant population in this region and since this type of precipitation is generally accepted as originating from the plasma sheet, it gives evidence for a near-Earth location of the inner edge of the plasma sheet in late growth phase.

Whenever it is possible to classify the spectra properly in the poleward precipitation region, a superposition of warm and cold populations is seen. If we assume that this precipitation is actually confined to a (possibly subvisual) arc which is the boundary of plasmas with different temperatures and densities, then it is interesting that plasmas with such different temperatures are adjacent and partially mixing with each other in the magnetosphere. To achieve a somewhat more quantitative idea whether this is reasonable, we used a global MHD/ionosphere coupling simulation (Janhunen, 1996) to produce a typical onset. The onset occurred at $X=-10, Y=-3$ in the model. The ionospheric footprints of field lines mapping to the vicinity of the onset region just before onset were then determined. It was found that the distance between open field lines and dipolar closed field lines in the onset sector was only $0.16^{\circ}$, i.e. about $16 \mathrm{~km}$ in the ionosphere. Although the numbers extracted from the simulation cannot be exact (we did not use any eventspecific solar wind data in this run, for instance), it is nevertheless interesting to see that the MHD simulation predicts that the plasma sheet precipitation region may become extremely narrow just before onset and that the distance in the ionosphere is of the same order of magnitude as the latitudinal extent of the poleward precipitation region seen in this study.

Acknowledgements. The Freja project was supported by the Swedish National Space Board (SNSB) and by the Deutsche Agentur für Raumfahrtangelegenheiten (DARA). The Freja satellite is managed and operated by the Swedish Space Corporation. We also thank the Kiruna group for providing the $\mathrm{F} 3 \mathrm{H}$ data and the F7 group for providing electron data. M. A. L. Persson, J. H. Clemmons, M. H. Boehm and R. A. Hoffman are gratefully acknowledged for helpful discussions and comments.

\section{References}

Arnoldy, R. L., T. E. Moore, and L. J. Cahill, Low-altitude fieldaligned electrons, J. Geophs. Res., 90, 8445- 8460, 1985.

Boehm, M. G., Paschmann, J. Clemmons, H. Höfner, R. Frenzel, M. Ertl, G. Haerendel, P. Hill, H. Lauche, L. Eliasson, and R. Lundin, The TESP electron spectrometer and correlator (F7) on Freja, Space, Sci. Rev., 70, 509-540, 1994.

Elphinstone, R. D., J. S. Murphree and L. L. Cogger, what is a global auroral storm, Rev. Geophys., 34, 169-232, 1996

Evans, D. S., Precipitating electron fluxes formed by a magnetic field aligned potential differences, J. Geophys. Res., 79, 28532858, 1974.

Frank, L. A., and K. L. Ackerson, Observations of charged particle precipitation into the auroral zone, J. Geophys. Res., 76, 3612, 1971.

Goertz, C. K., and R. W. Boswell, Magnetosphere-ionosphere coupling, J. Geophys. Res., 84, 7239-7246, 1979.

Hui, C. H., and C. E. Seyler, Electron acceleration by Alfvén waves in the magnetosphere, J. Geophys. Res., 97, 3953-3963, 1992.

Janhunen, P., T. I. Pulkkinen, and K. Kauristie, Auroral fading in ionosphere-magnetosphere coupling model: implications for possible mechanisms, Geophys. Res. Lett., 22, 2049-2052, 1995.

Janhunen, P., GUMICS-3 a global ionosphere-magnetosphere coupling simulation with high ionospheric resolution, Proc. of ESA 1996 symposium on environment modelling for space-based applications, ESA/ESTEC, 18-20, September-1996, ESA SP392, 1996.

Lemaire, J. and M. Scherer, Field-aligned current density versus electric potential characteristics for magnetospheric flux tubes, Ann. Geophysicae, 1, 91-96, 1983.

Lin, C. S., and R. A. Hoffman, Characteristics of the inverted-V event, J. Geophys. Res., 84, 1514-1524, 1979.

Lundin, R., G. Haerendel, and S. Grahn, The Freja science mission, Space. Sci. Rev., 70, 405-419, 1994. 
McFadden, J. P., C. W. Carlson, M. H. Boehm, and T. J. Hallinan, Field- aligned electron flux oscillations that produce flickering aurora, J. Geophys. Res., 92, 11,133-11,148, 1987.

McFadden, J. P., C. W. Carlson, and M. H. Boehm, Structure of an energetic narrow discrete arc, J. Geophys. Res., 95, 6533- 6547, 1990.

Olsson A., A. I. Eriksson and P. Janhunen, On the current-voltage relationship in westward travelling surges and auroral breakups, Ann. Geophysicae, 14, 1265-1273, 1996.
Persson, M. A. L., H. J. Opgenoorth, T. I. Pulkkinen, A. I. Eriksson, P. O. Dovner, G. D. Reeves, R. D. Belian, M. André, L. G. Blomberg, R. E. Erlandson, M. H. Boehm, A. T. Aikio, and I. Häggstrom, Near-earth substorm onset: a coordinated study, Geophys. Res. Lett., 21, 1875-1878, 1994. 